\title{
Ionospheric disturbances generated by different natural processes and by human activity in Earth plasma environment
}

\author{
HANNA ROTHKAEHL $\left({ }^{1}\right)$, NATALY IZOHKINA $\left({ }^{2}\right)$, IGOR PRUTENSKY $\left({ }^{2}\right)$, SERGEY PULINETS $\left({ }^{2}\right)\left({ }^{3}\right)$, \\ MICHEL PARROT $\left({ }^{4}\right)$, GEORGYI LIZUNOV $\left({ }^{5}\right)$, JAN BLECKI $\left({ }^{1}\right)$ and IWONA STANISLAWSKA $\left({ }^{1}\right)$ \\ (') Space Research Centre, Warsaw, Poland \\ $\left.{ }^{(}\right)$Institute of Terrestrial Magnetism, Ionosphere and Radio Wave Propagatio (IZMIRAN), \\ Russian Academy of Sciences, Troitsk (Moscow Region), Russia \\ ${ }^{3}{ }^{3}$ Instituto de Geofísica, UNAM, Ciudad Uni, Delegación de Coyoacán, México D.F. \\ (4) LPCE/CNRS, Orleans, France \\ $\left({ }^{5}\right)$ Kyiv Shevchenko University, Ukraine
}

The magnetosphere-ionosphere-thermosphere subsystem is strongly coupled via the electric field, particle precipitation, heat flows and small scale interaction. Satellites in situ measurements and ground based complex diagnostics can provide comprehensive coverage of both time and geomagnetic place effects. Human activity also can perturb Earth's environment, but few are connected with controlled experiments in the ionosphere and are transient. Most of them are related to industrial activity and have increased in recent years. The most important power sources are broadcasting transmitters, power stations, power lines and heavy industry. At ionospheric altitude some disturbances and physical processes are related to seismic activity, thunderstorm activity and some global changes in the Earth environment such as ozone holes. Various natural and artificial indicators can affect satellite telecommunication quality. The aim of this presentation is to report progress in understanding the physical processes in the ionosphere described above and to assess the application of these considerations to the study of plasma effects on Earth-space and satellite-to-satellite communication.

\subsection{ELECTROMAGNETIC AND ELECTROSTATIC INSTABILITIES DRIVEN BY CHANGES GEOMAGNETICS ACTIVITY IN THE NEAREST EARTH ENVIRONMENT}

To develop a quantitative model of the interaction of solar energetic particle events with Earth's magnetosphere it is necessary to consider plasma as well as wave diagnostics of space plasma. Despite a long history of ionospheric space investigations, the physical properties of ionospheric plasma are still not well understood. The global description of electromagnetic fields during the variable parameters in the Sun-Earth system is very difficult to do in the upper ionosphere. Natural plasma emission has become an object of interest since it was first received on the orbit of spacecraft. It is very hard to judge which physical process dominates during a geomagnetic disturbance, in particular in particular it seems necessary to bind the observations from low and high altitude together for a better understanding of the physical processes in the magnetosphere and ionosphere and to build a useful physical model for Space Wether Service purposes. 
In addition to passive information like local plasma density near space vehicles, wave diagnostics located on board spacecraft also give a dynamic view of plasma behaviour and instabilities. The HFwaves activity manifest in the ionosphere, can describe the property of local electron plasma and can also help to understand the property of remote plasma region. This frequency range covers high frequency whistler waves, Langmuir and upper hybrid modes of natural plasma, whereas non-linear plasma iteration can appear as a wide band turbulent spectrum.

The region of the main ionospheric trough acts like a lens focusing a variety of instabilities and disturbances from a different region of the Earth's environment. The mid-latitude electron density trough observed in the topside ionosphere has been shown to be the near-Earth signature of the magnetospheric plasmapause and thus its behaviour can provide useful information on magnetosphere dynamics since its existence is dependent on magnetospherically induced motions (Tulunay et al., 2003). The ionospheric trough is an unique region of the ionosphere, where different type of wave and instabilities can be generated.

Dynamic changes in the post-midnight outer ionosphere during an intense magnetic storm with SSC have been analysed using wave and plasma measurements (Rothkaehl et al., 1997). The investigation shows that HF and VLF wide band frequency emissions are good indicators of a turbulent
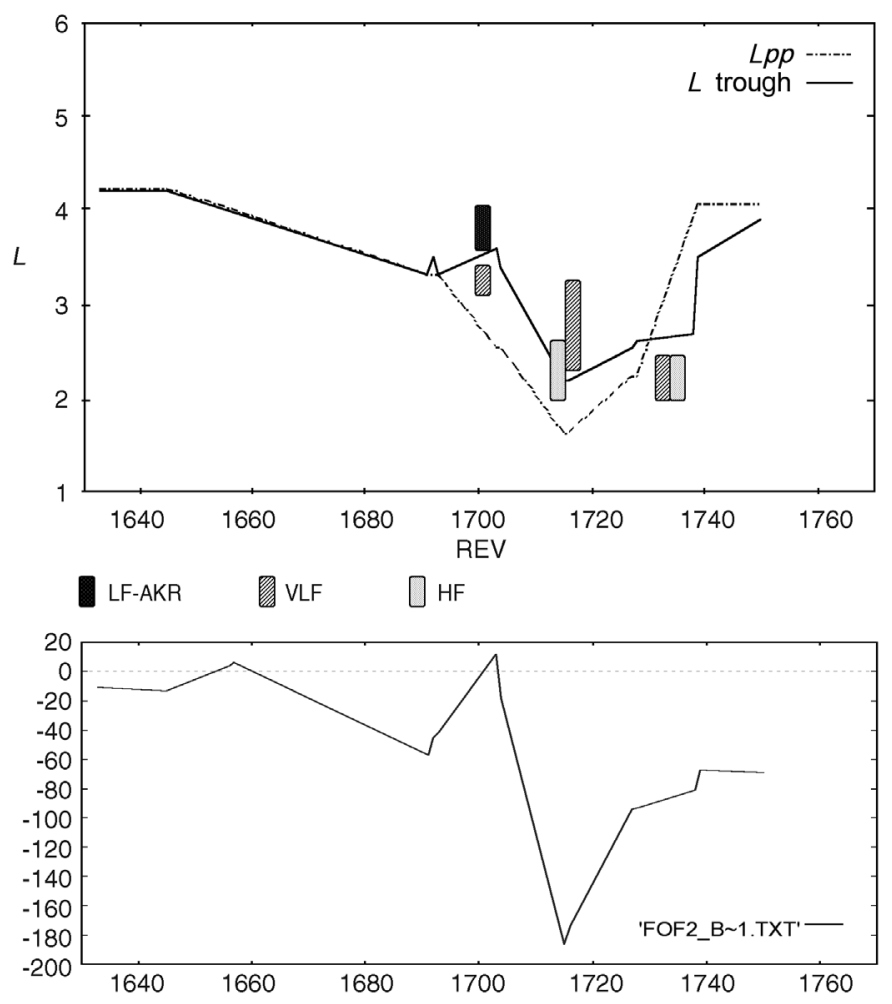

Fig. 17.1. The theoretical position of plasmapause Lpp, (solid line), and the position of the trough determined from the HF measurements versus sequence of satellite orbits (dashed line) with typical emissions during strong geomagnetic storm close to the plasmapause position, bottom panel the evolution of Dst index. 
plasma region closely related to the ionospheric trough features. Figure 17.1 presents the position of minimum mail ionospheric trough and typical waves activity. The detected VLF emission follows the quarter-gyrofrequency very well while HF radio emission indicates the enhancement of radiation at frequenciesy larger than the local upper hybrid limit. This broad band emission is mainly detected very close to the plasmapause, inside the plasmasphere but also inside the trough up to the auroral oval. This type of emission is mainly correlated to low frequency radiation, which is excite by the wave-particle interaction in the equatorial plasmapause and moves to the ionosphere along the geomagnetic field line. At the ionosphere the subthermal electron can interact with those electrostatic waves and excite the electron acoustic waves or the HF longitudinal plasma waves.

Fossil broad band emission was also detected inside the plasmosphere close to the plasmopause after strong geomagnetic disturbances. This type of emissions permit us to construct a more realistic model of the transfer energy in the ionosphere-magnetosphere coupling processes and to describe the region of plasma instability in the top side ionosphere.

Satellite in situ waves and plasma measurements and theoretical considerations yielded a spatial picture of this ionospherice region. It was shown that the peeling of plasma cloud associated with spatially nonuniform plasma from the low latitude trough wall during enhanced geomagnetic activity can be associated with plasma heating by electrostatic instability and atmospheric acoustic gravity waves generated in the auroral zone and damped in the ionosphere (Izovkina et al., 1999).

The polar cusp-cleft is a very sensitive region of the Earth environment for changes in geomagnetic condition and is one of the regions, where the most intense electrostatic and electromagnetic waves are generated. During reconnection at magnetopause in the cusp vicinity plasma particles are accelerated and plasma particle fluxes are generated.

Wave diagnostics located on board a low altitude orbiting satellite have demonstrated that during strong geomagnetic storms the cusp and cleft region is characterised by the appearance of: whistler mode, upper hybrid, electron plasma waves and lower hybrid resonance, as well as electron acoustic plasma waves. The observed emissions at low frequency and VLF frequency range are localised in space and have burst character and can be correlated with the filamentary structure of the cusp caused by current instabilities. The broad band HF whistler waves are good indicators of cusp positions particularly during strong geomagnetic disturbances. Wave particle interactions are proposed as a mechanism for the energy cascade from low frequency to high frequency waves. The discussed process has a self-consistent character and is accompanied by the heating and acceleration of particles in the polar cusp. Disturbances in the magnetopause can produce ion beams, which give rise to Alfven waves. As a consequence Alfven waves accelerate electrons. On the other hand, lower hybrid waves are also sources of energetic electrons. Theoretical consideration shows that the high frequency Langmuir waves can be excited by the beam of non thermal electrons or are driven by lower hybrid resonance. The most intensive waves in the cusp are related to the turbulent plasma region (Blecki et al., 2003).

The impact of magnetospheric disturbances on the ionospheric daily foF 2 frequencies is remarkable and can be closely related to the cusp feature (fig. 17.2). The geographical position of the cusp determined by high-latitude topside measurements seems to be very close to the disturbance seen in the foF 2 maps obtained from the PLES (Stanisławska and Rothkaehl, 2002) model even with some doubts concerning the mapping accuracy and topside data interpretations. In spite of these limitations, the almost real-time availability of both VI and topside measurements makes it possible to use them for the practical improvement of radio communication maps. Now the topside measurements can be treated not only as an indicator of the high altitude local disturbance that statistically will influence the electron concentration below, but as a physical source of redistribution of the electron concentration that will give exact figures when using simpler models. Future works will refine the dayside cusp-cleft model for radio communication purposes (Rothkaehl et al., 2003).

Large-scale spatial structures of electrostatic noise distribution observed near the geomagnetic equator can be related to variations in plasma dispersion parameters in the inhomogeneous magnetic field, the decay of electrostatic modes over the magnetic equatorial surface, and the possible formation of 

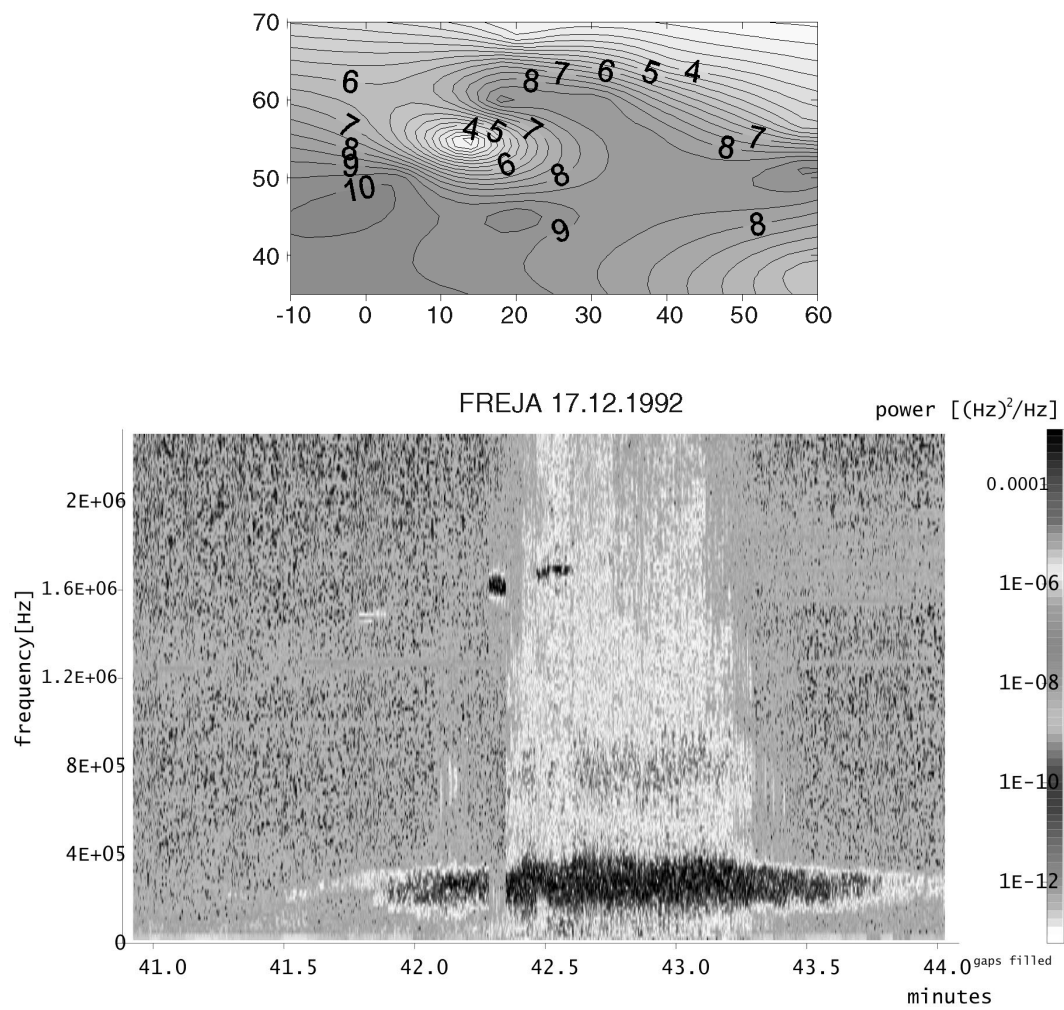

Fig. 17.2. FoF2 instantaneous map (MHz) for the European area for December 17, 199214 UT constructed during a geomagnetic storm (upper panel) and wave measurements display of the cusp region over the Atlantic Ocean seen from on board the Freja satellite 17.12.1992 at 18 UT (lower panel).

31.03.1994

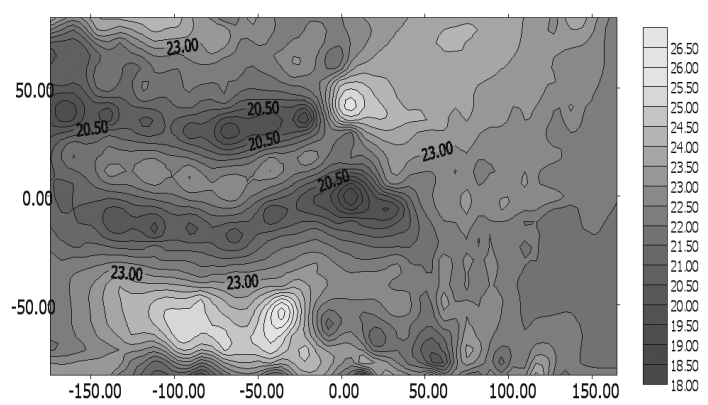

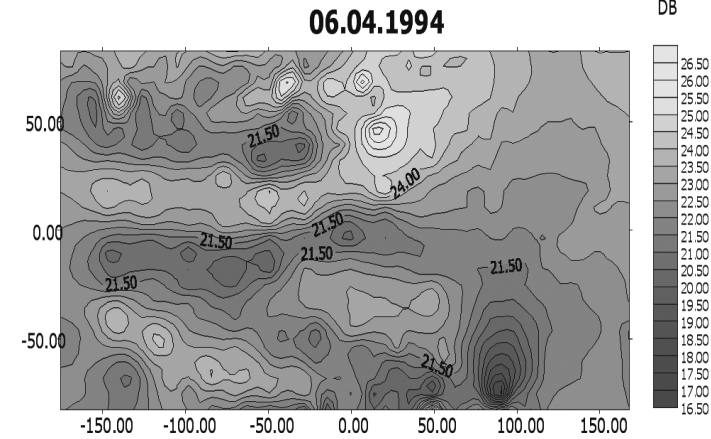

Fig. 17.3. Global distribution of HF emission in the ionosphere in the frequency range $0.1-2 \mathrm{MHz}$ during day time sector. The spectral intensity was integrated at around $500 \mathrm{~km}$ altitude 31.03 .1994 during quiet conditions and on 6.04.1994 during strong geomagnetic disturbances, recorded by the SORS-1 instrument on board the Coronas-I satellite. The well manifested enhancements of HF frequency (whistler frequency range) follow the geomagnetic equator and some enhancements are also remarkable in the region where energetic proton fluxes are detected. 
plasma vortices (with dimensions exceeding the ion Larmor radius) on density and temperature gradients of plasma. If a vortex propagates in the region of the inhomogeneous geomagnetic field, it can break up when the direction of the geomagnetic field vector at the equator becomes opposite. The formation of plasma vortices favors conservation of plasma inhomogeneities and consequently their detection. The appearance of additional broadband noise can be the manifestation of quasi-entrapment of electromagnetic waves between plasma walls of an equatorial cavern (Izovkina et al., 2002) (fig. 17.3).

The enhancement of broad band emissions in the electron whistler frequency band near equatorial equator is a common feature of global distribution natural electromagnetic noises in the ionosphere. This emission is associated with distribution of energetic particles from the radiation belt region.

\subsection{ELECTROMAGNETIC AND ELECTROSTATIC INSTABILITIES DRIVEN BY MAN-MADE INFLUENCES}

The ionosphere is modified unintentionally by various types of man-made noise. As a consequence electromagnetic emissions detected on board low orbiting satellites and propagated signals are affected by artificial influences. Perturbations of the nearest environment are due to different types of waves emitted from the ground and release of different gases into the atmosphere. A large variety of observed phenomena connected with human activity have been detected and analysed such as: wave particle interaction, precipitation of radiation belt electrons, parametric coupling of electromagnetic whistler waves, triggering emissions, frequency shifting and wave spectrum broadening. Most of these disturbances were observed at lower frequencies and only a few were correlated with the disturbances of HF electromagnetic emissions.

Some ground-based experiments were performed and consisted in controlled heating of the ionosphere by dense powerful electromagnetic waves. Synchronous detection of HF waves on board the Coronas-I satellite over SURA heating facility exhibited enhancement of background radiation parallel to the appearance of radiation at the 3rd and 5th order of electron cyclotron harmonics (Klos et al., 1997). Latest experimental results from ionosphere HF pumping experiments in the night-side auroral region above Troms $\varnothing$, show the triggering of local auroral activation. The authors suggest that these changes in the local ionospheric plasma are parallel to the appearance of the Alfven turbulence and the formation of the local magnetospheric current system.

The property of natural emissions depends very much of the conditions of the Sun's activity, but the influence of artificial disturbances is complex and very often relate to the weave particle and wave weave plasma interaction. We can observe parallel to direct radiation also in the emissions of ionos-

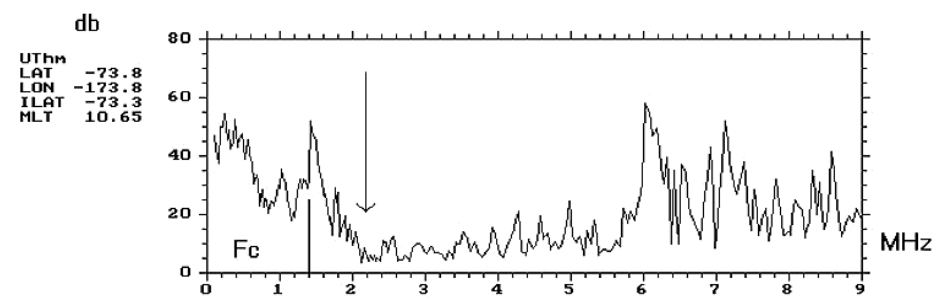

Fig. 17.4. The example of typical single HF spectrum detected on board the Coronas-I satellite, the line marks the local electron gyrofrequency at the satellite altitude and the arrow the cut-off of local plasma emission UHR (Upper Hybrid Resonance). The well pronounced emission for frequencies larger than UHR are related to human activity, mainly broadcasting station activity. 
06.04.1992

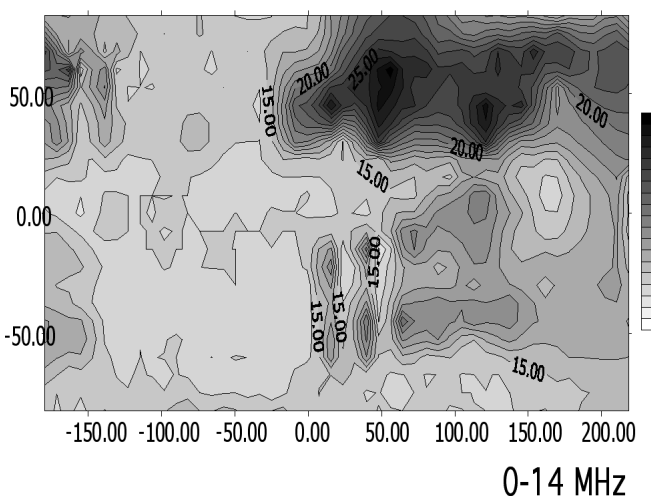

\subsubsection{2}

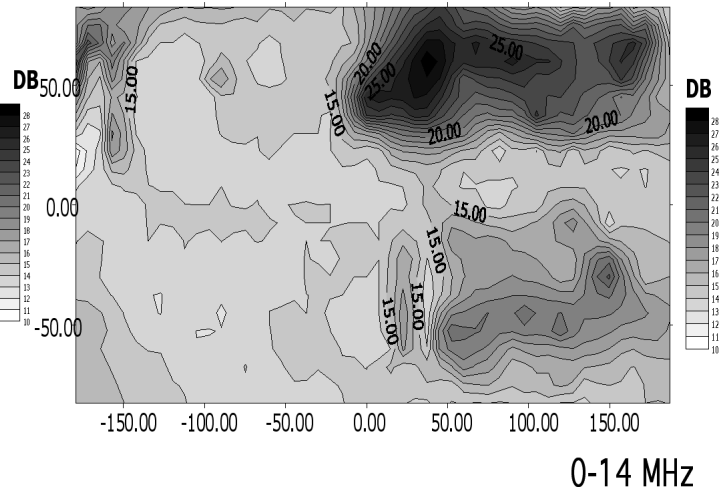

Fig. 17.5. The global distribution of mean value of electromagnetic emission in the ionosphere in the frequency range 0.1-15 MHz. The spectral intensity was integrated at various times of day and night 30.03.1994 during quiet condition and on 6.04.1994 during strong geomagnetic disturbances, recorded by SORS-1 instrument on board the Coronas-I satellite. The resolution is $5^{\circ} \times 5^{\circ}$; the units are $\mathrm{DB} / \mu \mathrm{V}$.

Eh

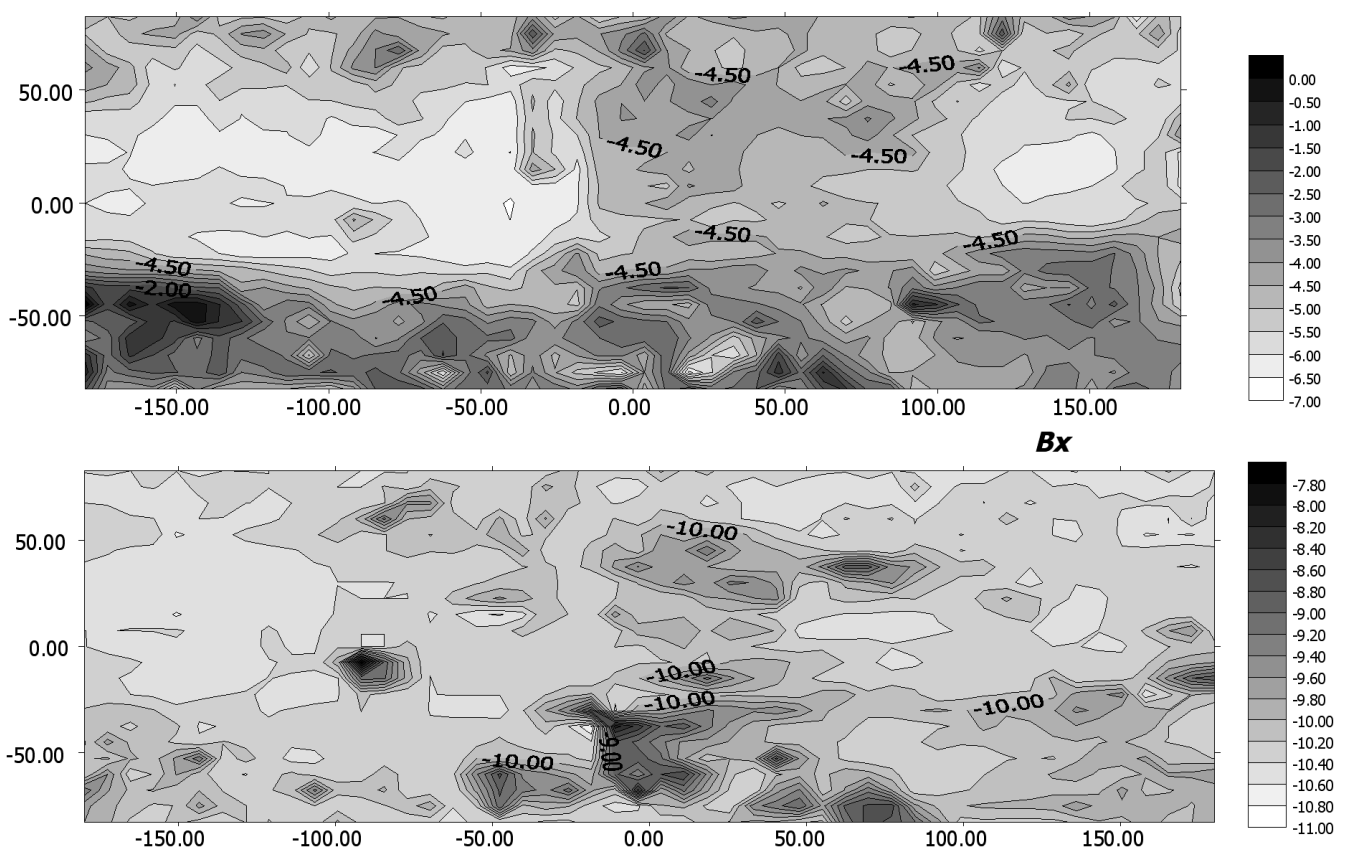

Fig. 17.6. The global distribution of the electric component $E h$ and magnetic component $B x$ recorded during very quiet condition from 11 to 13 January 1982 on board the AUREOL-3 satellite in the wide frequency band from 9 to $16 \mathrm{kHz}$. The intensity of detected emissions is in $\log (\mathrm{mV} / \mathrm{m} / \sqrt{ }(\mathrm{Hz}))$ for the electric component and $\log (\mathrm{nT} / \mathrm{m} / / \sqrt{ }(\mathrm{Hz}))$ for the magnetic component. 
pheric plasma triggered by transmitters of electromagnetic waves located around the world. Human activity can be seen on the recorded spectra as separated peaks, growing of the whole background of plasma emission or enhanced broadband emissions in the whole observed frequency range (fig. 17.4).

The permanently pumped electromagnetic waves to the ionosphere by the system of broadcasting stations can disturb the nearest space environment. Thus this activity can create in the topside ionosphere local Langmuir turbulence or ion-acoustic turbulence. However theoretical assumptions show that the generation of ion-acoustic turbulence is far more effective. The scattering of super-thermal electrons on ion-acoustic or Langmuir turbulence is proposed as a mechanism of generation of broad-band HF emissions. The HF diagnostics, performed on the low orbiting satellite, detected an enhancement of radiation particularly over the Euro-Asia region. HF emission in the topside ionosphere is more intense over the area correlated with outer radiation belts. It is prominent on the Eastern Hemisphere i.e. some longitudinal effect is manifested. The observed effect is markedly visible, and is stronger in the night-side ionosphere. Strong geomagnetic disturbances can broaden the area of observed intensification of HF noises in comparison with quiet time, but the intensity of the observed enhancement is not affected by geomagnetic disturbances (Rothkaehl and Klos, 2002) (fig. 17.5).

The VLF frequency emissions range is in principal related to the direct radiation of broadcasting station, high voltage power line and thunderstorm activity, but, the complex non-linear physical processes in ionospheric plasma described above should also change the nearest Earth environment. The examination of the data collected on the ARCAD-3 satellite helps us to find the emissions in the VLF frequency range correlated with broadband HF emissions detected over Europe and Asia region. Detailed examination of global distribution VLF emission detected in the topside ionosphere seems to be very complex for analysis due to propagation effects. The VLF natural plasma emissions are made by different types of natural and artificial origin noises. An effect of particle precipitation on natural whistler and hiss emissions as well as enhancement of made man noises triggered by VLF transmitters, Power-Line Harmonic Radiation PLHR and enhancement the VLF emissions related to thunderstorm activity have been shows (Parrot, 1990). They also concluded that maximum VLF hiss over North America is related to enhancements of thunderstorm activity region and PLHR emissions.

The presented example (fig. 17.6) shows that during very quiet condition we can detected the enhancements of VLF emission over Europe contrary to the more disturbed condition where the maximum is detected over regions of North America. It is important to underline that the described increase was detected during night time at altitudes larger than $1000 \mathrm{~km}$ during quiet thunderstorm activity.

\subsection{EARTHQUAKE ELECTROMAGNETIC SIGNATURES}

Many observational facts suggest the connection of earthquakes with disturbances of electromagnetic fields (LF VLF HF range) and associated phenomena. The statistical investigations of ELF/VLF emissions recorded by low orbiting altitude satellite AUREOL-3 done during seismic events are presented in the fig. 17.7 (Parrot, 1994). The correlation between seismic activity and wave enhancement shows that the extension of increase is in latitude but not in longitude (the $\Delta \operatorname{long}<10^{\circ}$ ). This indicates that, due to the propagation wave related seismic processes can be observed all along the magnetic meridian passing the epicentre of the earthquake.

Changes in the nearest Earth environment over seismic activity regions observed by the "Atmospheric Explorer - E» satellite and by the system of ground-based vertical ionosondes network have been investigated recently. Densities and temperature of neutral components $\mathrm{O}, \mathrm{N}_{2}$; main ion component $\mathrm{O}^{+}$ and densities of minor ionised components $\mathrm{NO}^{+}, \mathrm{O}^{+}{ }_{2}$ recorded on board the AE-E satellite as well as typical ionospheric parameters of $E$ - and $F$-layers recorded by VI measurements were analysed. The crosscorrelating spectra analysis of wavelength from $100 \mathrm{~km}$ to $2000 \mathrm{~km}$ displayed the enhancement of Atmospheric Gravity Waves, AGW, wavelike ion variations and Travelling Ionospheric Disturbances, TID. 


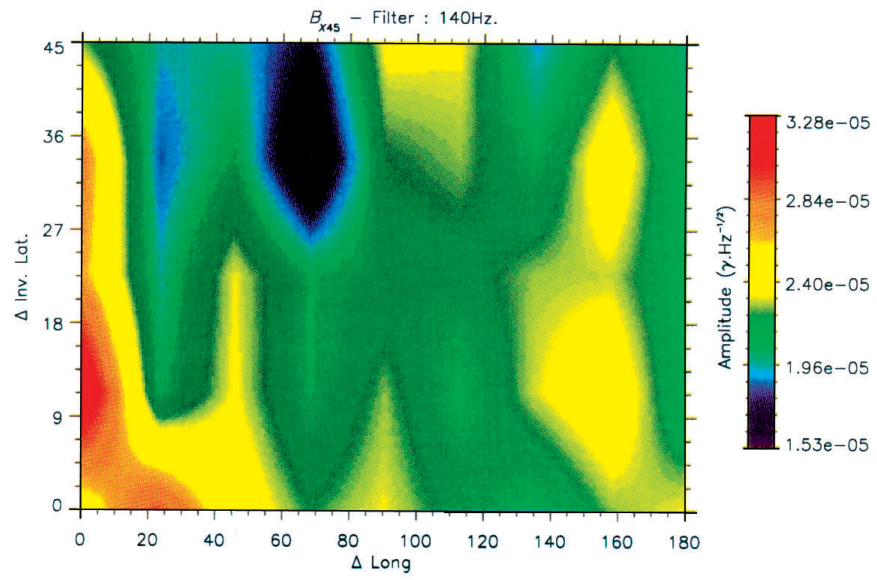

Fig. 17.7. The average amplitude of $140 \mathrm{~Hz}$ recorded by magnetic component during $24 \mathrm{~h}$ window on board the AUREOL-3 satellite, around 325 earthquakes with $M_{\mathrm{s}}>5$.

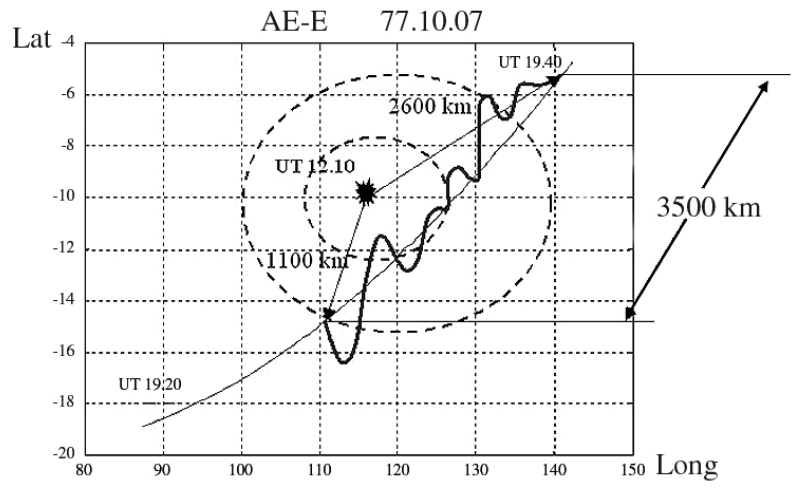

Fig. 17.8. Variation of ion concentration $\mathrm{O}^{+}$along LT, 77.10.07. The satellite pass perturbed region through $5 \mathrm{~h}$ after an earthquake, distant from the earthquakes epicentre $R(103 \mathrm{~km})$, detected on board the AE-E satellite at altitudes of up to several thousand $\mathrm{km}$ from epicenter.

Regular features of recorded variations appeared to be connected with solar, moon atmospheric tides, and terminator motion. Sporadic variations are caused by solar and geomagnetic activity as well as AGW penetrating the thermosphere «from below». Characteristic parameters of AGW/TID features generated by strong earthquakes $(M=7)$ at satellite altitudes (near $300 \mathrm{~km}$ ) have horizontal wavelength near $600 \mathrm{~km}$, while the amplitude of neutral density wave (AGW) reaches several percents, variations of ion density wave TIDs are stronger, reaching tens percent (fig. 17.8).

Observational effects can be explained in terms of AGW-ionosphere interaction. The hypothetical chain of events is as follows: earthquake shock can generate wave packet AGW waves propagating upward, than due to the filtering of large-scale AGW by the atmosphere we can observe heating of the upper atmosphere and the generation of TIDs. As a consequence we can detect the electric field irregularities. 
The physical picture of the relation between electromagnetic disturbances on the ground as well as at the ionospheric altitudes is not clear and more theoretical studies are needed. In the near future (April 2004) the French satellite DEMETER will be launched into orbit and its main goal will be to search for ionospheric effects such as disturbances of the electromagnetic field, plasma density, electron temperature, ion composition and temperature, Alfven, whistler and ion-acoustic waves associated with earthquakes.

\subsection{THE INTERACTION BETWEEN THE BODY OF SATELLITE AND SURROUNDING IONOSPHERIC PLASMA}

The level of electromagnetic radio noises observed on board satellites strongly depends on the properties of the satellite environment, as well as geophysical conditions. Behind the body of spacecraft the region of strong disturbed plasma is well manifested. The study of plasma wakes behind satellites has a long history in the investigation of Earth environment. The mesothermal motion of a satellite in the ionosphere creates a disturbed region behind the spacecraft that is depleted unequally of both ions and electrons. This type of irregularity has been mainly investigated by accurate measurements of electron density in the wake region while an enhancement of electron temperature has been observed in the discussed region. It is well known that for a small body the wake depletion varies approximately linearly, while for a large body the variation is exponential. The existence of excitation of ion acoustic waves behind a small satellite has been well examined. Also the unexpected broadband electrostatic emission behind large objects like Spacelab-2 has been observed and considered. The radiospectrometer observations recorded on board the Intecosmos19 and other plasma waves experiments like Active and Apex show some broad band emission in the HF frequency range. The measurements also show the enhancement of radiation between the local plasma frequency and critical frequency $f_{o F}$. It has been shown that the origin of this radiation can be correlated with the interaction between oscillation of electrostatically tapped particles in the wake of spacecraft and superthermal electrons (Rothkaehl, 1993).

The potential of spacecraft body surrounding in ionospheric or magnetospheric plasma can play a crucial role in understanding the electromagnetic stability of payload and antenna systems. A number of experiments have employed the artificial particle beams form ionospheric satellite to study various ionospheric and magnetospheric phenomena. These studies were used to diagnose auroral plasma properties and also to research the disturbances of propagated electromagnetic signal from one satellite to another. Theoretical considerations and data analysis of satellite experiments indicated that the charging of the satellite body by a dense electron beam strongly depends on the local electron density and in cases when local plasma is not sufficiently dense to neutralize electron current the overpotential takes place. The interaction of the injected beam with payload periodical potential can create strong wide band HF emission up to $80 \mathrm{MHz}$ (Zbyszyński, 2002).

\subsection{Conclusions}

Electromagnetic radiation in hecto and decametric range in the nearest space environment is a topic of investigations both as a constituent of the geophysical environment, and as an element of physical processes in which particles and waves of ionospheric plasma participate. Those investigations are lead using ground-based techniques and space borne instruments. The level of electromagnetic noise detected at ionospheric altitude depends on properties of geophysical condition, properties of the different Earth regions, influences from the Earth surface and the satellite surrounding plasma interaction. Human activity can perturb Earth's environment, but it does not have a direct impact, so the investigations should cover the wide band subject of physical processes in space. 
The various natural and artificial indicators can affect satellite telecommunication quality. The natural ionospheric radiation range around characteristic plasma emission in HF is of the order of $10^{-10} \mathrm{Wm}^{-2} \mathrm{~Hz}^{-2}$, while noises related solar radio emissions are of the order of $10^{-16} \mathrm{Wm}^{-2} \mathrm{~Hz}^{-2}$. Theoretical considerations show that the emitted power related to the human activity is in order of $10^{-18}$ $\mathrm{Wm}^{-2} \mathrm{~Hz}^{-1}$. The broad band emission detected in the ionosphere enriches the frequency bandwidth by a few MHz. This type of broad band emissions can influence on the global electromagnetic environment. The presented considerations can be used to construct global signal to noises maps used for telecommunication purposes.

Theoretical considerations of the physical property of ionospheric plasma bring us to a description of the spatial and temporary distribution of instabilities and disturbances generated in the nearest space environments. It has been shown that broad band emissions are related to the natural plasma instabilities driven by geomagnetic activity, some unintentional heating ionosphere by human activity, interaction between the satellite body and surrounding plasma and some catastrophic events on the Earth surface. The different types of ionospheric plasma instabilities described above can in consequence produce different types of irregularities both in ionisation and neutral plasma component. The aim of this presentation was to listen to the typical instabilities and regions, where different types of plasma irregularities can be recognized. The process described above can significantly influence propagation properties. Signal quality is affected by irregularities and instabilities in the ionosphere which produces phase and amplitude variations in the received signal. An in-depth understanding of these physical processes is helpful for telecommunication purposes.

\section{REFERENCES}

Blecki, J., S. Savin, N. Cornilleau-Wehrlin, M. Parrot, H.Rothkaehl, K. Stasiewicz, R. Wronowski, O. SANTOLIK and J.-A. SAUVAud (2003): Fine structure of the polar cusp as deducted from the plasma wave and plasma measurements, Adv. Space Res., 32 (3), 315-322.

Izhovkina, N.I., V.V. Afonin, A.T. KARPACheV, I.S. PRUTENSKII and S.A. Pulinets (1999): Structure of the ionospheric trough for different geomagnetic disturbance levels and the sources of plasma heating in the upper day side ionosphere, Magn. Aeron., 39 (4).

Izhovkina, N.I., I.S. Prutensky, S.A. Pulinets, Z. Klos and H. RothKaehl (2002): Electrostatic emissions in magnetoplasma irregularity in the region of the South Atlantic geomagnetic anomaly, Magn. Aeron., 42 (5), 660-665.

Klos, Z., H. Rothkaehl, Z. Zbyszyński, N.I Budko, I.S. Prutensky, S.A. Pulinets and V.V. VASKOV (1997): The modification of HF emissions in the upper ionosphere during the heating experiments over SURA facility, in Plasma 97, Research and Applications of Plasmas, edited by M. SADOWSKI and H. RothKAEHL, vol. 1, 379-383.

PARrot, M. (1990): World map of ELF/VLF emissions as observed by low-orbiting satellite, Ann. Geophysicae, 8, 135-146.

PARROT, M. (1994): Statistical study of ELF/VLF emissions recorded by a lowaltitude satellite during seismic events, J. Geophys. Res., 99 (A12), 23339-23347.

RothKAEHL, H. (1993): The HF noises related to the satellite wake in ionospheric plasma, Adv. Space Res., 13 (10), 309-312.

RothkAEHL, H. and Z. KLos (2002): Broadband HF emissions as an indicator of global changes within the ionosphere, Adv. Space Res., 31 (5), 1371-1376.

RothKAEHL, H., F. JIřIČEK, J. SMMILAUER and M. FÖRSTER (1997): Dynamic changes in the outer Ionosphere in the region of the ionospheric trough during an intense magnetic storm, Adv. Space Res., 20 (3), 409-414.

Rothkaehl, H., I. Stanistawska, J. Blecki and Z. ZbyszyŃski (2003): Low altitude cusp-cleft region signatures of strong geomagnetic disturbances, Cosmic Res., 41 (4), 340-344. 
Stanislawska, I. and H. Rothkaehl (2002): PLES model in the plasmapause diagnostics, Adv. Space Res., 29 (6), 833-838.

Tulunay, Y.K., I. Stanislawska and H. Rothkaehl (2003): Revisiting the Ariel trough work for HF telecommunication purposes, Cosmic Res., 41 (4), 319-331.

ZBYSZYŃSKI, Z. (2002): Spacecraft potential neutralization as a result of interaction ambient ionospheric plasma, on CD-Proceedings of the 1st COST 271 Workshop «Ionospheric Modelling and Variability Studies for Telecommunication Applications», RAL, 25-27 September 2001, edited by A. VERNON. 